\title{
Lebensqualitätsforschung bei Demenzkranken ist sinnlos
}

\author{
Research on Quality of Life doesn't Make Sense in Persons with Dementia
}

Autoren

Pro: Matthias Riepe

Kontra: Hans Gutzmann

Bibliografie

DOI http://dx.doi.org/

$10.1055 / \mathrm{s}-0030-1248414$

Psychiat Prax 2010; 37: e7-e10

(c) Georg Thieme Verlag KG

Stuttgart · New York .

ISSN 0303-4259

\section{Korrespondenzadressen \\ Prof. Dr. Matthias Riepe \\ Sektion Gerontopsychiatrie Psychiatrie II, Universität Ulm Bezirkskrankenhaus Günzburg Ludwig-Heilmeyer-Straße 2 89312 Günzburg \\ stefanie.vrba@bkh-guenzburg. de}

\section{Prof. Dr. Hans Gutzmann}

Klinik für Psychiatrie, Psychotherapie und Psychosomatik Krankenhaus Hedwigshöhe Höhensteig 1 12526 Berlin h.gutzmann@alexius.de

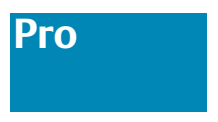

Es ist eine ärztliche Aufgabe, die Qualität des Lebens von Menschen mit Demenz mit den bestmöglichen pharmakologischen und nichtpharmakologischen Therapien, die in Studien der evidenzbasierten Medizin eine Wirksamkeit gezeigt haben, aufrechtzuerhalten oder zu verbessern. Die „Qualität des Lebens“ ist jedoch nicht gleichzusetzen mit „Lebensqualität“. Der Begriff der „Lebensqualität“ und die Lebensqualitätsforschung sind untrennbar mit konzeptuellen Vorstellungen konnotiert, die bei Demenzpatienten ihre Anwendbarkeit verlieren und in der Anwendung zu Konsequenzen führen, die dem Störungsbild der Alzheimer-Erkrankung und anderer Demenzen nicht gerecht werden.

\section{Das Konzept „Lebensqualität“}

In der wissenschaftlichen Literatur, v. a. der sozialwissenschaftlichen Literatur, erhält der Begriff „Lebensqualität“ seit den 70er-Jahren des vorigen Jahrhunderts Bedeutung. Es ist ein Konzept, das darauf abzielt, die individuellen Lebensumstände, Wahrnehmungen und Präferenzen aus der Sicht des Individuums zu erfassen. Entwickelt wurde dieses Konzept vor dem Hintergrund einer bis dahin überwiegend an den materiellen Ressourcen orientierten sozialwissenschaftlichen Forschung zu individuellen Lebensbedingungen. In Bezug auf Patienten fußt der Begriff außerdem auf der Definition dessen, was vonseiten der WHO unter Gesundheit verstanden wird, als einem Zustand des vollständigen körperlichen, geistigen und sozialen Wohlergehens und nicht nur dem Fehlen von Krankheit oder Gebrechen.

Historisch gesehen kommt der Begriff der Lebensqualität aus einer Zeit, in der die besonderen Herausforderungen der Demenzerkrankungen nicht berücksichtigt wurden, weil die wissenschaftliche Beschäftigung mit Demenzerkrankungen aufgrund des demografischen Wandels erst in den 80er-Jahren begann. Zwar wurden einflussreiche
Modelle, z. B. das von Lawton, in empirischen Untersuchungen auf Patienten mit der AlzheimerErkrankung übertragen [1]. Jedoch wurden die methodischen Grenzen der Selbsteinschätzung bei Patienten mit Erkrankungen des Zentralnervensystems nicht konzeptuell reflektiert auch wenn seit der konzeptuellen Formung des Begriffs „Lebensqualität“ zahlreiche Instrumente erprobt worden sind. Hierzu gehören z. B. der Euroquol (EQ-5d), die dementia quality of life scale (Demqol), die quality of life scale in Alzheimer's disease (QoL-AD), und die WHO-quality of life scale (WHOQOL) [2-6]. Zu jeder der genannten Skalen gehört die Selbsteinschätzung durch den Patienten. Der Umfang reicht von 5 Fragen beim EQ-5d bis zu mehr als 20 Fragen bei der DemqolSkala.

\section{Das klinische Bild des demenziellen \\ Syndroms}

Das demenzielle Syndrom ist nach der in Deutschland empfohlenen ICD-Klassifikation ein Syndrom, das aus Gedächtnisstörungen und weiteren kognitiven Einschränkungen (z. B. Orientierung, kognitive Flexibilität, exekutive Funktionen, Visuokonstruktion) besteht. Darüber hinaus tritt bei der Alzheimer-Erkrankung das gesamte Spektrum nichtkognitiver Symptome auf (z.B. Affekt, Agitation, Depression, Wahn). Die Symptome, die zur Diagnosestellung führen, sollen so stark ausgeprägt sein, dass sie zu einer beträchtlichen Beeinträchtigung im Alltag führen oder mit anderen Worten: die Qualität des Lebens im Alltag betreffen. In der Definition des Demenzsyndroms ist daher - anders als bei Erkrankungen, die primär histologisch (onkologische Erkrankungen) oder anhand von Laborparametern (Diabetes mellitus) charakterisiert werden - die Qualität des Lebens im Alltag bereits enthalten. 
Ergebnisse der Lebensqualitätsforschung bei Demenz Sowohl bei der Alzheimer-Demenz als auch bei einer Vorstufe, der milden kognitiven Beeinträchtigung, ist die Fähigkeit, die eigene Situation einzuschätzen, reduziert [7]. Der Grund für die zur Wirklichkeit disparaten Angaben des Patienten ist in der voranschreitenden Pathologie und der hierdurch bedingten Fehlfunktion vieler zentralnervöser Leistungen zu sehen, die bei Schätzvorgängen zu einfachen Alltagsaufgaben anfangen [8] und die kürzlich ausführlich diskutiert wurden [6]. Die Aussage, dass „Lebensqualität“ nicht durch den Patienten eingeschätzt werden kann, gründet nicht darauf, dass bisherige Instrumente unzureichend sind, sondern ist neurobiologisch begründet durch die schon in Prodromalstadien der Alzheimer-Demenz auftretende Fehlfunktion von neuronalen Regelkreisen.

Die Einschätzung der „Lebensqualität“ des Patienten durch den Betreuer ist keine Alternative. Sie ist nicht nur ein logischer Bruch zu dem Konzept der individuell eingeschätzten „Lebensqualität“, sie ist auch aus methodischen Gründen nicht möglich. Die Einschätzung der „Lebensqualität“ eines Demenzkranken durch einen Angehörigen ist abhängig von medizinischen und nichtmedizinischen Faktoren des Angehörigen [9] und bildet damit nicht nur die Qualität des Lebens der Patienten ab.

\section{Der ökonomische Aspekt der Lebensqualitätsforschung} Die notwendige Ökonomisierung der Medizin lässt Gesundheitsökonomen nach einem Maß rufen, das krankheitsübergreifend den Erfolg des Einsatzes von Ressourcen einschätzt. Um dies zu erreichen, sollen Erkrankungen miteinander verglichen werden, die unterschiedliche Organsysteme betreffen, die in verschiedenen Lebensaltern auftreten, die einmalig oder chronisch auftreten, die rezidivierend oder progredient sind. Nur ein ubiquitäres Instrument könnte methodisch sauber eine Vergleichbarkeit von Krankheitslast und Interventionserfolg herstellen. Einfache und kurze Instrumente wie der EQ-5D sind daher im Umfeld gesundheitsökonomischer Diskussionen beliebt. Hier zeigt sich aber besonders deutlich, dass schon die Auswahl der abgefragten items der „Lebensqualität“ das Ergebnis beeinflusst. Bei der EQ-5DSkala findet sich je eine Frage zu Beweglichkeit/Mobilität, zu der Fähigkeit, für sich selbst zu sorgen, zu alltäglichen Aktivitäten, zu Schmerzen/körperlichen Beschwerden sowie zu Angst/ Niedergeschlagenheit. Die Beeinträchtigung der kognitiven Leistungsfähigkeit z. B. taucht hier gar nicht auf, obwohl sie die personale Identität des Menschen sicher noch stärker betrifft als die Mobilität. Auch die Psyche des Menschen tritt mit dieser Auswahl an Items, wie ja an vielen anderen Stellen in der Gesellschaft auch, zurück hinter den Aspekt der Körperlichkeit.

Die Antworten in Fragebögen zur „Lebensqualität“ und die erneute Einschätzung nach Anwendung einer medikamentösen oder nichtmedikamentösen Intervention dienen zunehmend als Grundlage dafür, zusammen mit dem Preis einer Intervention die Kosteneffektivität zu berechnen. Lebensqualitätsforschung bei Demenzerkrankungen trägt daher zu einer Vorenthaltung therapeutischer Ressourcen bei, weil aufgrund der krankheitsbedingten Einschränkungen keine Schweregradabhängigkeit der Lebensqualitätseinschätzung durch den Patienten besteht obwohl eine Schweregradabhängigkeit der alltagsrelevanten Beeinträchtigungen, der kognitiven Defizite und der Verhaltenssymptome, also der Qualität des Lebens von Demenzerkrankten, besteht.
"Lebensqualität" und neuropsychiatrische Gesundheit ein Beispiel

Der Mann der ehemaligen Richterin am obersten Gerichtshof der USA, Sarah O'Connor, erkrankte an einer Demenz, die es trotz der Aufgabe des Berufs der Ehefrau im Verlauf erforderte, dass der Mann in ein Pflegeheim eingewiesen wurde, weil er seine Frau nicht mehr erkannte. Im Pflegeheim verliebte er sich in eine andere Frau, sodass man allem Anschein nach davon ausgehen konnte, dass er eine hohe „Lebensqualität“ hatte.

Aus ärztlicher und psychiatrischer Sicht muss allerdings hinzugefügt werden, dass nichtsdestotrotz der Patient seiner Biografie, seiner familiären Beziehungen, seiner Erinnerungen und vieler weiterer Dinge beraubt war, die sein Leben und seine personale Identität ausgemacht hatten. Es kann daher nicht das ärztliche Ziel sein, bei der Betrachtung der „Lebensqualität“ zu verharren, sondern es muss das Ziel sein, die Progredienz der Demenzerkrankungen zu verhindern und residuale Ressourcen und Potenziale für den Patienten nutzbar zu machen und, wenn möglich, einen Bezug zu seiner Biografie aufrechtzuerhalten. Die Voraussetzungen dafür können anhand biometrisch fundierter neuropsychologischer und neuropsychiatrischer Untersuchungsmethoden, die die Progredienz der Erkrankung und die Veränderung unter Therapie abbilden, eingeschätzt werden.

\section{Zusammenfassung und Schlussfolgerung}

Lebensqualitätsmessung durch Patienten mit Demenzerkrankungen ist aus neurobiologischen Gründen nicht möglich (vgl. [6]). Es ist und bleibt ärztliche Aufgabe, den Patienten und seine Situation einfühlend zu verstehen und die Auswirkungen von kognitiven Defiziten und Verhaltensstörungen im Alltag zu ermessen. Der Wunsch nach Messung der „Lebensqualität“ als patient-reported outcome ist Ausdruck einer Entbiologisierung des in der Psychiatrie ansonsten erfolgreichen biopsychosozialen Modells weil das Konzept „Lebensqualität“ neurobiologische Grundlagen der Beurteilung von Situationen und Fähigkeiten durch Demenzerkrankte missachtet. Lebensqualitätsforschung trägt damit bei zu einer Entärztlichung der Medizin. Lebensqualitätsforschung durch Messung als patient-reported outcome ist nicht nur sinnlos. Sie führt zu einer Missachtung der Qualität des Lebens von Patienten mit Demenzerkrankungen, der Potenziale von Erkrankten und der Therapiebedürftigkeit alltagsrelevanter Symptome. Lebensqualitätsmessung trägt so zu einer Minderversorgung der Patienten und Angehörigen bei.

\section{Kontra}

Die Lebensqualität der Patienten gilt zunehmend als eines der wichtigsten Kriterien zur Beurteilung der Effektivität therapeutischer Maßnahmen bei Demenzerkrankungen. Sie ist üblicherweise als eine multidimensionale Bewertung des Person-Umwelt-Systems konzeptualisiert, die sowohl objektive als auch subjektive Kriterien umfasst und durch die Komponenten psychisches Befinden, körperliche Gesundheit, soziale Beziehungen und funktionale Kompetenz zu charakterisieren ist. Als gängige methodische Zugänge gelten Verhaltensbeobachtung, Fremdauskunft und Befragung der Erkrankten. Nur der letztgenannte Zugang gibt die Chance, Auskunft über die subjektive Erfahrung der Befragten zu erhalten, zu denen im gegebenen Zusammenhang vornehmlich Lebenszufriedenheit und Wohlbefinden zählen. In unserem Zusammenhang mit der Demenz stellt sich weniger die Frage, ob die Ermittelung der Betroffenenperspektive angesichts der progredienten kognitiven Einbuße und einer schwindenden Krank- 
heitseinsicht möglich ist, als vielmehr auf welche Weise sie vorgenommen werden sollte.

Subjektive Gedächtnisstörungen können die Lebensqualität der nicht dementen Altenbevölkerung erheblich beeinträchtigen [10]. In Initialphasen von Demenzerkrankungen kann die einsetzende kognitive Beeinträchtigung ebenfalls als erhebliche Minderung der Lebensqualität erlebt werden. Im weiteren Verlauf ergibt sich in der Mehrzahl der Studien aber kein messbarer Zusammenhang mehr zwischen der Lebensqualität und dem Grad der kognitiven Einbusse, wohl aber mit dem Grad der Depressivität [11]. Als Begründung für diesen Befund wird auf eine zunehmende Anosognosie verwiesen [12]. Damit wird ein klinisches Zustandsbild bezeichnet, das durch eine beeinträchtigte oder fehlende Einsicht in krankheitsbedingte Defizite gekennzeichnet ist. Anosognosie ist nicht demenzspezifisch, auch bei Schizophrenien ist die Krankheitseinsicht oft vermindert. Obwohl für viele Demenzkranke eine mangelnde Krankheitseinsicht mit dem Voranschreiten des Prozesses positiv korreliert und sie zudem in der Betreuung erhebliche Probleme bereitet, ist der Forschungsstand noch unbefriedigend [12]. Differenzialdiagnostische Aspekte, etwa Unterschiede in der Selbsteinschätzung zwischen frontotemporalen Degenerationen und Alzheimer-Demenzen, scheinen in diesem Kontext weder hinsichtlich Gedächtnisstörungen noch im Bezug auf Verhaltensauffälligkeiten eine wesentliche Rolle zu spielen [13]. Während eine fehlende Krankheitseinsicht in späteren Krankheitsphasen enger mit hirnstrukturellen Schädigungen verknüpft ist, werden für Frühstadien psychologische Selbstschutzfunktionen diskutiert. Die Anosognosie soll danach, gleichsam als Bewältigungsstrategie, eine Selbstwertstabilisierung gewährleisten und gleichzeitig eine drohende Stigmatisierung ebenso abwehren wie eine Gefährdung der Autonomie des Individuums [14]. Die Patienten können demnach im Verlauf einer demenziellen Erkrankung sowohl durch neuropsychologische als auch psychische Mechanismen in der Wahrnehmung der eigenen Defizite beeinträchtigt werden. Die Differenzierung dieser Einflussgrößen ist schwierig, kann jedoch mit der Ermittlung von Krankheitsverarbeitungs- und -bewältigungsmustern erreicht werden [12]. Wenn Demenzkranke die eigene kognitive Leistungsfähigkeit systematisch überschätzen, die eigene Befindlichkeit dagegen adäquat einschätzen, scheint es plausibel zu sein, diese Leistungen zwei differenten Domänen zuzuordnen [11], die in unterschiedlichem Tempo und Umfang vom Krankheitsprozess betroffen werden. Studiendaten belegen, dass auch kognitiv schwerer beeinträchtigte Kranke eine valide Einschätzung ihrer Lebensqualität abgeben können, wenn nicht eine gravierend beeinträchtigte körperliche Gesundheit, eine ausgeprägte Apathie oder eine deutlichere depressive Symptomatik das Ergebnis verfälschen [15]. Diese Störvariablen lassen sich aber klinisch mit geeigneten Instrumenten erfassen. Zu berücksichtigen ist schließlich, dass die Selbsteinschätzung der Lebensqualität auch eine Funktion des eigenen Anspruchsniveaus ist, das unter den Bedingungen der Krankheit im Sinne eines Adaptationsprozesses eine deutliche Absenkung erfahren kann [16]. Wenn auf diesem Wege das Anspruchsniveau des Patienten und das seiner Angehörigen oder Betreuer zunehmend auseinander klaffen, wundert es nicht, dass deren Fremdeinschätzung der Lebensqualität regelmäßig unter denen der Patienten liegen. In gleicher Weise beeinflusst die eigene Belastung durch die Pflege die Einschätzung der Lebensqualität der Betreuten weit mehr als deren tatsächliche Alltagskompetenz [17]. Der Umstand, dass selbst bei gering ausgeprägter oder fehlender Anosognosie die Selbst- und Fremdeinschätzungen aus den genannten Gründen bedeutsam voneinander abweichen, legt den Gedanken nahe, dass die unterschiedlichen Einschätzungen zwei Perspektiven darstellen, die beide für sich potenziell valide sein können [18]. Die Validität der Selbsteinschätzung allein durch die Fremdeinschätzung bestimmen zu wollen, wie es häufig geschieht [17], erscheint unter diesem Aspekt wenig hilfreich. Die Ermittelung anderer Kenngrößen, etwa der internen Konsistenz [15] oder der konvergenten Validität durch Überprüfung der Übereinstimmung zwischen unterschiedlichen Selbsteinschätzungsinstrumenten, dürfte dagegen angemessener sein [19]. Wenn Lebensqualität als ein wesentlicher Outcome-Parameter angesehen wird, so muss davon ausgegangen werden, dass beim Selbstrating das Ausmaß der Depressivität wesentliche Anteile der Varianz erklärt. Der zusätzliche Einsatz eines entsprechenden Instruments zur Erfassung der Depressionsschwere gehört also zur Erhebung von Lebensqualität.

Die meisten Autoren stimmen darin überein, dass Patienten mit leichter bis mittelschwerer Demenz ihre Lebensqualität valide und reliabel einzuschätzen vermögen [17]. Mit fortschreitender Erkrankung wird dieses Urteil jedoch unzuverlässiger. Bei längsschnittlich angelegten Studien, in deren Verlauf die Probanden die Grenze verlässlicher Reliabilität zu überschreiten drohen, sollte die Selbsteinschätzung durch eine Fremdeinschätzung ergänzt werden. Dabei muss klar sein, dass dadurch ein substanzielles methodisches Problem aufgeworfen wird, das auch durch den Einsatz kombinierter Instrumente, die für frühe Phasen ein Selbstrating und bei Fortschreiten der Erkrankung ein Fremdrating vorsehen, nur unzulänglich behoben werden kann $[18,19]$. Die Applikation und Integration beider Sichtweisen zu Studienbeginn erscheint als ein möglicher Weg, die unmittelbare Sicht der Erkrankten so lange wie möglich systematisch Berücksichtigung finden zu lassen [15].

Lebensqualität lässt sich vom Demenzkranken, zumindest in leichteren Stadien, in zutreffender Weise in Erfahrung bringen. Zur Identifizierung und Ausschaltung von Störvariablen ist eine sorgfältige Auswahl des methodischen Zugangs und der einzusetzenden Instrumente erforderlich. Der mitunter vorgebrachte Einwand, die Auswirkungen der sich progredient entwickelnden Demenzsymptome machten eine valide Einschätzung von Lebensqualität kaum möglich, darf der Entwicklung einer sorgfältigen Konzeption zur Identifzierung von Lebensqualität bei diesem Personenkreis nicht im Wege stehen.

\section{Literatur}

1 Lawton MP. Quality of life in Alzheimer disease. Alzheimer Dis Assoc Disord 1994; 8 (Suppl. 3): 138-150

2 Banerjee S, Smith SC, Lamping DL et al. Quality of life in dementia: more than just cognition. An analysis of associations with quality of life in dementia. J Neurol Neurosurg Psychiatry 2006; 77: 146-148

3 Coons SJ, Rao S, Keininger DL et al. A comparative review of generic quality-of-life instruments. Pharmacoeconomics 2000; 17: 13-35

4 Khanna D, Tsevat J. Health-related quality of life - an introduction. Am J Manag Care 2007; 13 (Suppl. 9): S218-S223

5 Orley J, Saxena S, Herrman H. Quality of life and mental illness. Reflections from the perspective of the WHOQOL. Br J Psychiatry 1998; 172: 291-293

6 Riepe MW, Mittendorf T, Forstl $H$ et al. Quality of life as an outcome in Alzheimer's disease and other dementias - obstacles and goals. BMC Neurol 2009; 9: 47

7 Vogel A, Stokholm J, Gade A et al. Awareness of deficits in mild cognitive impairment and Alzheimer's disease: do MCI patients have impaired insight? Dement Geriatr Cogn Disord 2004; 17: 181-187

8 Barabassy A, Beinhoff U, Riepe MW. Cognitive estimation in mild Alzheimer's disease. J Neural Transm 2007; 114: 1479-1484

9 Karlawish JH, Casarett D, Klocinski J et al. The relationship between caregivers' global ratings of Alzheimer's disease patients' quality of life, 
disease severity, and the caregiving experience. J Am Geriatr Soc 2001; 49: 1066-1070

$10 \mathrm{Mol} \mathrm{M}$, Carpay M, Ramakers I et al. The effect of perceived forgetfulness on quality of life in older adults; a qualitative review. Int J Geriatr Psychiatry 2007; $22: 393-400$

11 Vogel A, Mortensen EL, Hasselbalch SG et al. Patient versus informant reported quality of life in the earliest phases of Alzheimer's disease. Int J Geriatr Psychiatry 2006; 21: 1132-1138

12 Leicht H, Gertz HJ. Methoden zur Erfassung von Krankheitseinsicht eine kritische Übersicht. Psychiat Prax 2009; 36: 58-66

13 Banks S, Weintraub S. Self-awareness and self-monitoring of cognitive and behavioral deficits in behavioral variant frontotemporal dementia, primary progressive aphasia and probable Alzheimer's disease. Brain Cogn 2008; 67: 58-68

14 Stechl E, Lämmler G, Steinhagen-Thiessen E et al. Subjektive Wahrnehmung und Bewältigung der Demenz im Frühstadium - SUWADEM. Z Gerontol Geriatr 2007; 40: 71-80
15 Hoe J, Katona C, Roch B et al. Use of the QOL-AD for measuring quality of life in people with severe dementia - the LASER-AD study. Age Ageing 2005; 34: 130-135

16 Angermeyer MC, Kilian R. Theoretical models of quality of life for mental disorders. In: Katschnig H, Freeman H, Sartorius N. Quality of life in mental disorders. Chichester: John Wiley \& sons, 2006: 21-32

17 Leipold B, Zank S. Die Erfassung subjektiver Lebensqualität bei demenziell Erkrankten: Gütekriterien von Selbstbeurteilungsskalen. Z Neuropsychol 2002; 13: 139-148

18 Ready RE, Ott BR, Grace J. Insight and cognitive impairment: effects on quality-of-life reports from mild cognitive impairment and Alzheimer's disease patients. Am J Alzheimers Dis Other Demen 2006; 21: $242-248$

19 Roick C, Hinz A, Gertz HJ. Kann Lebensqualität bei Demenzkranken valide bestimmt werden? Eine aktuelle Übersicht über Messinstrumente und methodische Probleme. Psychiat Prax 2007; 34: 108-116 